\title{
Effect of Polymyxin B Nonapeptide on Daptomycin Permeability and Cell Surface Properties in Pseudomonas aeruginosa, Escherichia coli, and Pasteurella multocida
}

\author{
Charles M. Morris ${ }^{\mathrm{a}}$, Abraham George ${ }^{\mathrm{b}}$, W. William Wilson ${ }^{\mathrm{b}}$ \\ and Franklin R. Champlin ${ }^{\mathrm{a}, *}$ \\ Departments of Biological Sciences ${ }^{a}$ and Chemistry $^{b}$, Mississippi State University, \\ P.O. Drawer GY, Mississippi State, MS 39762 U.S.A.
}

(Received for publication August 22, 1994)

\begin{abstract}
The present study was carried out to determine if sensitization of Gram-negative bacteria to the polyanionic antibiotic daptomycin by cationic molecules can be explained on the basis of decreased cell surface charge in order to better understand intrinsic resistance. Turbidimetric assessments of batch cultural growth kinetics revealed the outer membrane permeabilizer polymyxin B nonapeptide sensitized Pseudomonas aeruginosa and Escherichia coli to the hydrophobic probe novobiocin, whereas little or no sensitization was observed for two surface hydrophobicity variants of Pasteurella multocida. Polymyxin B nonapeptide and daptomycin synergistically inhibited growth of $P$. aeruginosa only. A hydrocarbon adherence assay revealed permeabilizing concentrations of polymyxin B nonapeptide increased cell surface hydrophobicity of $P$. aeruginosa and the hydrophobic $P$. multocida variant, while $E$. coli and the hydrophilic $P$. multocida variant remained unaffected. Measurement of cellular electrophoretic mobility showed polymyxin B nonapeptide permeabilization of $P$. aeruginosa to daptomycin occurred concomitantly with a significant decrease in cell surface charge, while no such sensitization occurred in organisms which failed to undergo polymyxin B nonapeptide-induced surface charge decreases. These data suggest that sensitization of Gram-negative bacteria to polyanionic lipopeptides by growth in the presence of polycationic outer membrane permeabilizers such as polymyxin B nonapeptide is dependent on diminution of overall cell surface charge and polarity, thereby allowing outer cell envelope permeation.
\end{abstract}

Pseudomonas aeruginosa ${ }^{1)}$, Escherichia coli ${ }^{2)}$, and Pasteurella multocida ${ }^{3)}$ possess typically Gram-negative ultrastructure in that their cytoplasmic membrane and peptidoglycan components are surrounded by an outer membrane which functions in most such organisms as a diffusion barrier to lipophilic molecules ${ }^{4)}$. However, we have found that with the exception of the polyanionic lipopeptide daptomycin ${ }^{5}$, the outer membrane of naturally-occurring $P$. multocida strains is permeable to structurally-diverse hydrophobic antimicrobial agents ${ }^{6}$. That the basis for Gram-negative resistance to daptomycin is of a nonspecific nature and involves outer membrane impermeability is strongly suggested by its exclusively Gram-positive antibacterial spectrum ${ }^{7)}$, despite possessing a primary mechanism of action which appears to involve the calcium-dependent depletion of cytoplasmic membrane energization ${ }^{8)}$ and inhibition of amino acid transport ${ }^{9}$.

We have previously speculated that in view of the general nature of cell envelope permeability to lipophilic molecules in $P$. multocida, intrinsic daptomycin resistance may be due to electrostatic repulsion between the negatively-charged outer membrane surface and the anionic antibiotic, thereby precluding their association ${ }^{6}$. LAKEY and LEA ${ }^{10}$ have suggested the inability of daptomycin analog A21978C to cause hemolysis may be due to the negatively-charged surface of erythrocytes. The assessment of net bacterial cell surface charge can be accomplished in a precise manner by determining cellular electrophoretic mobility ${ }^{11)}$.

CHAMPLIN and HART $^{5)}$ found polymyxin B (PMB) disruption of the outer membrane sensitized $P$. multocida P-1581, but not $P$. aeruginosa PAO1 or $P$. multocida ATCC 11039, to daptomycin, thereby suggesting the ability of the outer membrane to exclude daptomycin does not always require normal ultrastructural integrity. Polymyxin B nonapeptide (PMBN) is much less toxic to Gram-negative bacteria than PMB, yet retains the ability to permeabilize outer membranes ${ }^{12,13)}$. PETERSON et al. ${ }^{14)}$ showed that the polycationic PMB binds to lipopolysaccharide, thereby rigidifying it by altering its overall charge and perturbing the packaging arrangement of outer membrane components. PMBN lacks the PMB fatty acid tail while retaining its electrostatically positive nature. We employed PMBN in an effort to sensitize $P$. aeruginosa, $E$. coli, and $P$. multocida strains to 
daptomycin reasoning this compound might mitigate the negative charge of the cell surface while disorganizing the outer membrane such that daptomycin would penetrate in a manner not masked by inherent toxicity of the permeabilizer itself.

Resultant data provide further evidence that intrinsic daptomycin resistance in Gram-negative bacteria is in fact outer membrane mediated and normally intact structure is not essential to the ability of the outer membrane to preclude its permeation. Moreover, sensitization to polyanionic lipopeptides such as daptomycin by polycationic permeabilizers is dependent on the ability of the compounds to sufficiently decrease the net negative electrostatic charge of the outer membrane surface such that antibiotic association with and diffusion through the outer cell envelope can occur.

\section{Materials and Methods}

Bacterial Strains and Inocula Preparation

P. aeruginosa $\mathrm{PAO} 1$, E. coli ATCC 25922, P. multocida ATCC 11039 (hydrophilic cell surface variant) ${ }^{15)}$, and P. multocida P-1581 (hydrophobic cell surface variant) ${ }^{16)}$ were obtained ${ }^{6)}$ and maintained under cryoprotective conditions $^{16)}$ as described elsewhere. Stationary-phase inocula for all experiments were taken from starter cultures consisting of $20 \mathrm{ml}$ of Mueller-Hinton broth (MHB; Difco Laboratories, Detroit, MI) supplemented with $\mathrm{Ca}^{2+}\left(50 \mu \mathrm{g} / \mathrm{ml}\right.$ as $\left.\mathrm{CaCl}_{2} ; \mathrm{MHB}-\mathrm{Ca}\right)$ in $125-\mathrm{ml}$ growth flasks after overnight incubation ( $15 \sim 18$ hours) at $37^{\circ} \mathrm{C}$ with rotary aeration at $180 \mathrm{rpm}$ in a model G24 Environmental Incubator Shaker (New Brunswick Scientific Co. Inc., Edison, NJ). MHB-Ca was prepared by aseptically adding $5 \mathrm{ml}$ of filter-sterilized $(0.22-\mu \mathrm{m}$ Acrodisc disposable filter assemblies; Gelman Sciences, Inc., Ann Arbor, MI) $\mathrm{CaCl}_{2}$ solution $\left(3.68 \mathrm{~g}\right.$ of $\mathrm{CaCl}_{2}$. $2 \mathrm{H}_{2} \mathrm{O}$ per $100 \mathrm{ml}$ of $\mathrm{H}_{2} \mathrm{O}$ ) to one liter of sterile MHB.

\section{MIC Determination}

Stock solutions of the outer membrane permeabilizer PMBN (Boehringer Mannheim Biochemicals, Indianapolis, IN), and the antibiotic probes novobiocin sodium salt (control; Sigma Chemical Co., St. Louis, MO) and daptomycin (test; Lilly Research Laboratories, Eli Lilly and Co., Indianapolis, IN) were prepared in MHB-Ca, filter sterilized, and stored at $4^{\circ} \mathrm{C}$ for no more than seven days. Daptomycin potency was examined periodically by determining the MIC for daptomycin-susceptible Streptococcus agalactiae 090 (Dr. S. B. PruetT, Mississippi State University, Mississippi State, MS). MICs of all permeabilizer and probe molecules were determined using a standard macrobroth dilution method ${ }^{17)}$ as modified for use in this laboratory ${ }^{16)}$ with $\mathrm{MHB}-\mathrm{Ca}$ employed as diluent. Each dilution tube was inoculated with one volume of an early exponential-phase batch
MHB-Ca culture ( $c$ a. $2.5 \times 10^{5} \mathrm{cfu} / \mathrm{ml}$ final viable cell density) cultivated as described below.

\section{Experimental Batch Culture Conditions}

Batch cultures were grown in 125-ml flasks containing $50 \mathrm{ml}$ of MHB-Ca each (flask: volume ratio of 2.5) after inoculation with stationary-phase starter cultures to an initial optical density of approx. 0.05 at $550 \mathrm{~nm}\left(\mathrm{OD}_{550}\right.$; Spectronic 20D Optical Spectrophotometer; Milton Roy, Co., Rochester, NY). Cultures were incubated at $37^{\circ} \mathrm{C}$ with rotary aeration until early exponential phase $\left(\mathrm{OD}_{550}\right.$ of 0.10$)$, at which time $2.5-\mathrm{ml}$ aliquots were dispensed into test culture tubes $(18 \times 150 \mathrm{~mm}$; Kimax $)$ containing $2.5 \mathrm{ml}$ of $\mathrm{MHB}-\mathrm{Ca}$ (control), or MHB-Ca supplemented with sublethal concentrations of PMBN, novobiocin, daptomycin, PMBN plus novobiocin, or PMBN plus daptomycin and incubated as before in order to determine the effect of growth in the presence of PMBN on outer cell surface permeability, hydrophobicity, and electrostatic charge.

\section{Outer Membrane Perturbation Assay}

The ability of novobiocin or daptomycin to synergistically inhibit the growth of test organisms in the presence of PMBN was assessed on the basis of turbidimetric growth kinetic measurements using the method of CHAMPLIN and HART ${ }^{5)}$.

\section{Cell Surface Hydrophobicity Assay}

Cell surface hydrophobic properties of control and PMBN-treated cells were assessed on the basis of the degree to which they partitioned into $n$-hexadecane ${ }^{18)}$. Cells from late exponential-phase test cultures $\left(\mathrm{OD}_{550}\right.$ of approximately 0.50 ) were pooled, harvested by centrifugation at $10,000 \times g$ and $4^{\circ} \mathrm{C}$ for 10 minutes, washed with one volume of cold PPMS buffer $\left(6.97 \mathrm{~g}\right.$ of $\mathrm{K}_{2} \mathrm{HPO}_{4}$, $2.99 \mathrm{~g}$ of $\mathrm{KH}_{2} \mathrm{PO}_{4}$ and $0.2 \mathrm{~g}$ of $\mathrm{MgSO}_{4} \cdot 7 \mathrm{H}_{2} \mathrm{O}$ per liter of glass-distilled water; $\mathrm{pH} 7.12$ ), and suspended in room temperature PPMS buffer to an $\mathrm{OD}_{550}$ of 0.50 . Cell surface hydrophobicity was measured as described previously ${ }^{16)}$.

\section{Cell Surface Charge Assay}

Cell surface electrostatic charge properties of control and PMBN-treated cells were assessed by measuring electrophoretic mobility of cultures in terms of zeta potential $^{11)}$ as determined on the basis of light scattering analysis using a Coulter DELSA 440 (Coulter Corporation, Hialeah, FL). Late exponential-phase test cultures $\left(\mathrm{OD}_{550}\right.$ of $\left.c a .0 .50\right)$ were placed in an ice bath until ready for analysis.

\section{Statistical Analysis}

Numerical data were analyzed for statistical significance by employing the Student $t$ test and the two tailed table to determine $P$ values. $P$ values less than 0.01 and 0.05 indicate differences between means are statistically significant at the $99 \%$ and $95 \%$ confidence 
levels, respectively.

\section{Results}

Susceptibility to Outer Membrane Permeabilizers and Antibiotic Probes

The MICs of permeabilizer and probe molecules for all test organisms can be seen in Table 1. The greater than ten-fold decrease in MIC exhibited by daptomycin for $S$. agalactiae in $\mathrm{MHB}-\mathrm{Ca}^{2+}$ confirms the $\mathrm{Ca}^{2+}$ dependency of its mechanism of action reported elsewhere for various Gram-positive bacteria ${ }^{7,8)}$. The decision was made to incorporate $\mathrm{Ca}^{2+}$ in all experimental media in order to optimize the bacteriocidal properties of daptomycin, thereby increasing the sensitivity of the cell envelope permeability assay. Data obtained using the hydrophobic cell surface variant $P$. multocida $\mathrm{P}-1581$ reveal that, unlike the parent compound PMB, PMBN is nonlethal in the presence of added $\mathrm{Ca}^{2+}$. All test organisms were clearly resistant to both PMBN and daptomycin. In contrast, only $E$. coli ATCC 25922 and $P$. aeruginosa PAO1 were resistant to novobiocin, while $P$. multocida strains ATCC 11039 and P-1581 were susceptible as previously reported ${ }^{6)}$.

\section{Effect of PMBN on Outer Membrane Permeability Properties}

We have previously employed turbidimetric assessments of cultural growth kinetics to determine that PMB is able to nonspecifically permeabilize the outer membrane of $P$. multocida $\mathrm{P}-1581$, thereby sensitizing it to daptomycin in $\mathrm{MHB}^{5)}$. A similar degree of PMBinduced sensitization to daptomycin was obtained more

Table 1. Susceptibility of organisms to polymyxin B, polymyxin B nonapeptide, novobiocin, and daptomycin as determined by broth dilution assay in MHB-Ca.

\begin{tabular}{lccrc}
\hline \multirow{2}{*}{ Organism } & \multicolumn{4}{c}{ MIC $(\mu \mathrm{g} / \mathrm{ml})^{\mathrm{a}}$} \\
\cline { 2 - 5 } & PMB & PMBN & NOV & DAP \\
\hline P. aeruginosa PAO1 & ND & $>256.0$ & $>256.0$ & $>256.0$ \\
E. coli ATCC 25922 & ND & $>256.0$ & 128.0 & $>256.0$ \\
$P$. multocida & ND & $>256.0$ & 4.0 & $>256.0$ \\
$\quad$ ATCC 11039 & & & & \\
$P$. multocida P-1581 & 2.0 & $>256.0$ & 2.0 & $>256.0$ \\
$S$. agalactiae 090 & ND & ND & ND & $0.25(8.0)^{\mathrm{b}}$ \\
\hline
\end{tabular}

a Each value was obtained from at least three replicate two-fold serial dilutions using a macrobroth dilution assay in MHB supplemented with $\mathrm{Ca}^{2+}(50 \mu \mathrm{g} / \mathrm{ml})$. Abbreviations: PMB, polymyxin B; PMBN, polymyxin B nonapeptide; NOV, novobiocin; DAP, daptomycin; ND, not determined.

b Assay performed in MHB without $\mathrm{Ca}^{2+}$ supplementation. recently in $\mathrm{MHB}$ containing $\mathrm{Ca}^{2+}$ at the concentration employed for the present study (unpublished data). The nonbacteriocidal $\mathrm{PMBN}^{13}$ ) was selected for subsequent use as a permeabilizing agent under these conditions after it was found to render $P$. aeruginosa PAOl susceptible to novobiocin, while poly-L-lysine, EDTA, and diaminoacetone exhibited only slight to moderate synergy with novobiocin under these conditions ${ }^{19)}$.

Control experiments were performed to establish the degree to which PMBN is able to nonspecifically permeabilize the outer membranes of test organisms as judged on the basis of sensitization to the normally excluded hydrophobic antibiotic novobiocin. As can be seen in Fig. 1, growth in the presence of PMBN significantly sensitized $P$. aeruginosa PAO1 and $E$. coli ATCC 11039 to novobiocin, while little or no synergy was observed in the $P$. multocida variants. It should be noted that growth of the $P$. multocida variants was consistently less in the presence of both PMBN and

Fig. 1. Growth of test organisms in the presence of PMBN and/or NOV. Each value represents the mean of three to six independent determinations.

(A) $P$. aeruginosa PAO1; Symbols: $O$, control; $\bullet$, PMBN $(10 \mu \mathrm{g} / \mathrm{ml}) ; \square$, NOV $(256 \mu \mathrm{g} / \mathrm{ml})$; and $\mathbf{e}$, PMBN $(10 \mu \mathrm{g} / \mathrm{ml})$ and NOV $(256 \mu \mathrm{g} / \mathrm{ml})$. (B) E. coli ATCC 25922; Symbols: $O$, control; $\bullet, \operatorname{PMBN}(8 \mu \mathrm{g} / \mathrm{ml}) ; \square$, NOV $(4 \mu \mathrm{g} / \mathrm{ml})$; and $\mathbf{n}$, PMBN $(8 \mu \mathrm{g} / \mathrm{ml})$ and NOV $(4 \mu \mathrm{g} / \mathrm{ml})$. (C) P. multocida ATCC 11039; Symbols: $O$, control; $\bullet$, PMBN (128 $\mu \mathrm{g} / \mathrm{ml})$; $\square, \operatorname{NOV}(1 \mu \mathrm{g} / \mathrm{ml})$; and $\mathbf{n}$, PMBN $(128 \mu \mathrm{g} / \mathrm{ml})$ and NOV $(1 \mu \mathrm{g} / \mathrm{ml})$. (D) P. multocida P-1581; Symbols: $O$, control: $\bullet$, PMBN $(10 \mu \mathrm{g} / \mathrm{ml}) ; \square, \operatorname{NOV}(0.5 \mu \mathrm{g} / \mathrm{ml})$; and $\square$, PMBN $(10 \mu \mathrm{g} / \mathrm{ml})$ and NOV $(0.5 \mu \mathrm{g} / \mathrm{ml})$.

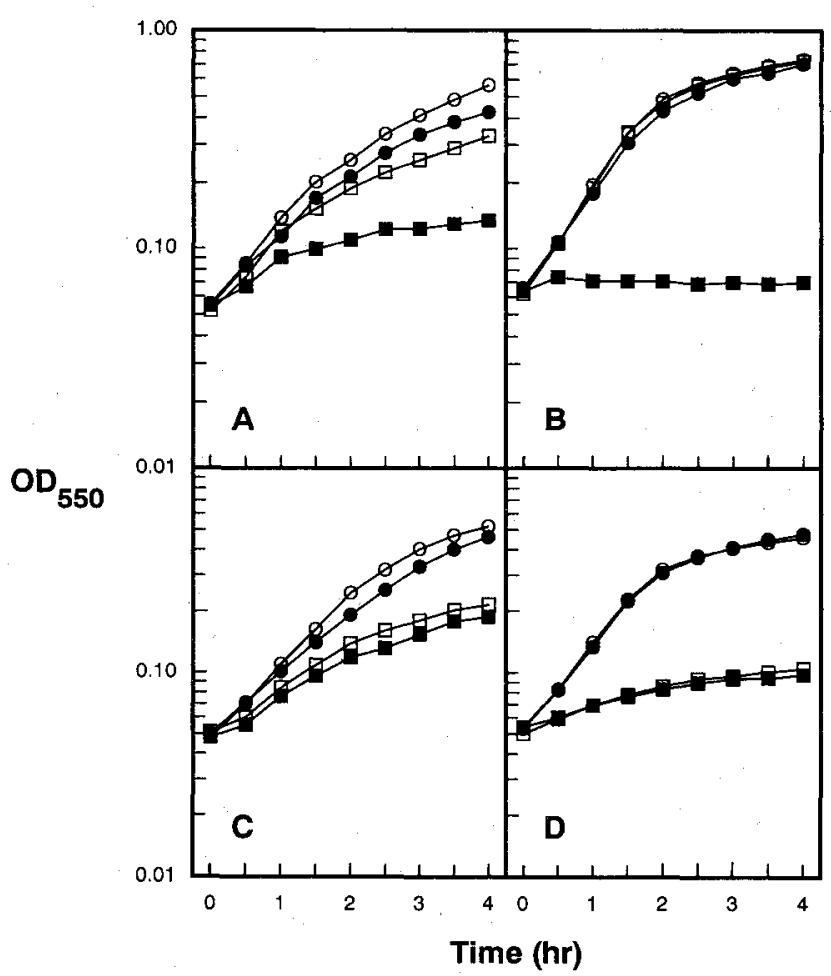


novobiocin than that seen with novobiocin alone in each of up to six independent determinations. It therefore appears likely that a slight, yet reproducible, potentiation of novobiocin growth inhibition does occur for this organism in the presence of PMBN. Data in Fig. 2 reveal PMBN also sensitized $P$. aeruginosa PAO1 to

Fig. 2. Growth of test organisms in the presence of PMBN and/or DAP. Each value represents the mean of three to four independent determinations.

(A) P. aeruginosa PAO1; Symbols: $O$, control; $\bullet$, PMBN $(10 \mu \mathrm{g} / \mathrm{ml}) ; \square$, DAP $(400 \mu \mathrm{g} / \mathrm{ml}) ;$ and $\mathbf{m}$, PMBN $(10 \mu \mathrm{g} / \mathrm{ml})$ and DAP $(400 \mu \mathrm{g} / \mathrm{ml})$. (B) E. coli ATCC 25922 ; Symbols: $\mathrm{O}$, control; $\bullet$ PMBN $(8 \mu \mathrm{g} / \mathrm{ml}) ; \square$, DAP $(400 \mu \mathrm{g} / \mathrm{ml})$; and $\mathbf{\square}$, PMBN $(8 \mu \mathrm{g} / \mathrm{ml})$ and DAP $(400 \mu \mathrm{g} / \mathrm{ml})$. (C) P. multocida ATCC 11039; Symbols: $\bigcirc$, control; •, PMBN (128 $\mu \mathrm{g} / \mathrm{ml})$; $\square$, DAP $(400 \mu \mathrm{g} / \mathrm{ml})$; and $\boldsymbol{\Xi}$, PMBN $(128 \mu \mathrm{g} / \mathrm{ml})$ and DAP (400 $\mu \mathrm{g} / \mathrm{ml})$. (D) P. multocida P-1581; Symbols: O, control; $\bullet$ PMBN $(10 \mu \mathrm{g} / \mathrm{ml}) ; \square$, DAP $(400 \mu \mathrm{g} / \mathrm{ml})$; and $\mathbf{\square}$, PMBN $(10 \mu \mathrm{g} / \mathrm{ml})$ and DAP $(400 \mu \mathrm{g} / \mathrm{ml})$.

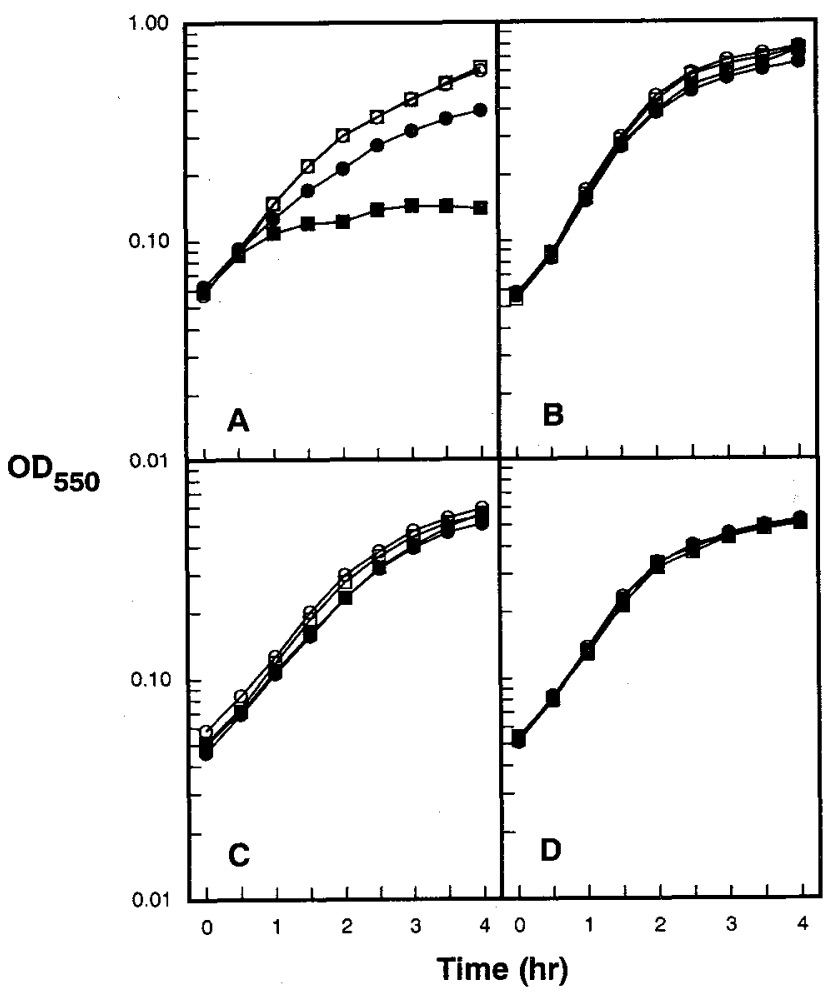

daptomycin, while all other organisms remained unaffected.

\section{Cell Surface Hydrophobicity}

The relative abilities of control and PMBN-treated cells to associate with $n$-hexadecane were examined in order to determine if PMBN-induced permeabilization is accompanied by a concomitant change in overall cell surface hydrophobicity. Growth in the presence of PMBN at concentrations employed in attempts to permeabilize outer membranes to daptomycin caused significant increases in cell surface hydrophobicity of $P$. aeruginosa PAO1 and the hydrophobic $P$. multocida variant P-1581 (Table 2), even though only the former organism was sensitized to the antibiotic (Fig. 2). No significant differences in hydrophobicity properties were detected between treated and untreated $E$. coli ATCC 29522 and $P$. multocida ATCC 11039.

\section{Cell Surface Charge}

In order to determine the effect of PMBN-induced permeabilization on cell surface charge, zeta potential values reflective of the degree of net surface electronegativity were determined for test organisms grown in the absence and presence of the permeabilizer. $P$. aeruginosa PAO1 underwent a significant decrease in overall negative cell surface charge as a result of being cultured in the presence of PMBN (Table 2). In contrast, PMBN exerted no significant effect on the cell surface charge properties of $E$. coli ATCC 25922, P. multocida ATCC 11039, or P. multocida P-1581.

\section{Discussion}

The exclusively Gram-positive antibacterial spectrum of daptomycin suggests the intrinsic resistance exhibited by Gram-negative organisms is nonspecific and most likely involves outer membrane impermeability. Failure of the outer membrane to function as a barrier to the

Table 2. Effect of PMBN permeabilization on cell surface hydrophobicity and surface charge.

\begin{tabular}{|c|c|c|c|c|c|c|}
\hline \multirow{2}{*}{ Organism } & \multicolumn{3}{|c|}{ Percent adherence to hexadecane ${ }^{a}$} & \multicolumn{3}{|c|}{ Zeta potential ${ }^{\mathbf{b}}$} \\
\hline & Control & Treated $^{\mathrm{c}}$ & $P$ value & Control & Treated $^{\mathfrak{c}}$ & $P$ value \\
\hline$P$. aeruginosa $\mathrm{PAO} 1$ & $-0.68 \pm 0.38$ & $8.12 \pm 0.68$ & $<0.05$ & $-24.50 \pm 1.82$ & $-13.18 \pm 2.03$ & $<0.01$ \\
\hline E. coli ATCC 25922 & $2.30 \pm 1.45$ & $0.60 \pm 1.93$ & $>0.05$ & $-30.15 \pm 4.35$ & $-30.36 \pm 4.74$ & $>0.01$ \\
\hline P. multocida ATCC 11039 & $-0.69 \pm 0.49$ & $0.35 \pm 0.67$ & $>0.05$ & $-26.65 \pm 5.85$ & $-22.91 \pm 7.67$ & $>0.01$ \\
\hline P. multocida $\mathrm{P}-1581$ & $30.43 \pm 4.77$ & $58.20 \pm 5.24$ & $<0.05$ & $-10.07 \pm 4.31$ & $-9.34 \pm 8.57$ & $>0.01$ \\
\hline
\end{tabular}

a Percent decrease in turbidity of standardized cell suspension after mixing with $1,000 \mu \mathrm{l}$ of $n$-hexadecane. Each value represents the mean of three-to-four independent determinations.

$\mathrm{b}$ Zeta potential of standardized cell suspension expressed in $\mathrm{mV}$. Each value represents the mean of four-to-six independent determinations.

c Cells grown in the presence of PMBN at the concentrations used in attempts to sensitize respective organisms to NOV and DAP. 
diffusion of antimicrobial agents can be examined by sensitization of bacteria to otherwise excluded molecules as assessed on the basis of turbidimetric measurements of altered cultural growth kinetics ${ }^{5,20}$. CHAMPLIN and HART $^{5)}$ employed this method to determine the outer membranes of $P$. aeruginosa PAO1, $P$. multocida ATCC 11039 (a hydrophilic cell surface variant), and $P$. multocida P-1581 (a hydrophobic cell surface variant) were rendered permeable to the hydrophobic antibiotic novobiocin by growth in the presence of sublethal concentrations of either EDTA or PMB. Furthermore, PMB sensitized P. multocida P-1581 to daptomycin. The failure to sensitize $P$. aeruginosa PAO1 and P. multocida ATCC 11039 to daptomycin under conditions wherein their outer membranes were otherwise permeabilized to novobiocin suggests the ability of the outer cell envelope to retard daptomycin diffusion does not require normally intact structure. That $P$. multocida hydrophobic variant P-1581 was rendered susceptible to daptomycin by PMB under conditions which sensitized all organisms to novobiocin supports the hypothesis that intrinsic daptomycin resistance is a result of the permeability barrier function of the outer membrane. Furthermore, otherwise permeabilized outer membrane surfaces may retain sufficient residual negative charge to preclude association with the polyanionic daptomycin due to electrostatic repulsion in certain cases. PMB treatment caused no detectable changes in cell envelope lipid composition or cell surface charge (as judged using electrostatic interaction chromatography) of the $P$. multocida strains, thereby leaving strain variation with regard to susceptibility to PMB-daptomycin synergy unexplained. However, this study was hampered by the facts that permeabilizing concentrations of PMB are very close to bacteriocidal levels ${ }^{13)}$ and a much more reliable method for measuring cell surface charge is available ${ }^{111}$.

The net negative surface charge of Gram-negative bacteria is due in large part to the presence of lipopolysaccharide in the outer leaflet of the outer membrane ${ }^{21)}$. The negative surface properties of $P$. multocida serotype A variants, as represented in this study by strain ATCC 11039 , are most likely enhanced by capsular material which is composed largely of hyaluronic acid ${ }^{22)}$. The relative cell surface hydrophobicity of $P$. multocida variants has been shown to be related to the degree to which they are encapsulated and the hyaluronidase removal of capsular material is accompanied by a concomitant enhancement of hydrophobic properties ${ }^{15}$.

We reasoned it should then be possible to sensitize $P$. aeruginosa and $E$. coli (both having outer membranes refractory to hydrophobic molecules), and further sensitize P. multocida (which has an outer membrane permeable to hydrophobic molecules) to daptomycin by exposing them to the nonbacteriocidal outer membrane permeabilizer PMBN in the presence of added $\mathrm{Ca}^{2+}$. Different amounts of synergy between PMBN and novobiocin for the various organisms indicate that PMBN compromises the ability of their respective outer membranes to function as permeability barriers to lipophilic molecules to different degrees. That $P$. aeruginosa PAO1 was rendered susceptible to daptomycin by PMBN-induced outer membrane permeabilization is strongly supportive of the hypothesis that intrinsic daptomycin resistance in Gram-negative bacteria is a function of the outer membrane.

The inability of PMBN to sensitize the remaining organisms to daptomycin, while also failing to decrease their respective cell surface electronegativity, supports the hypothesis that sensitization by PMB and PMBN is dependent on their abilities to obviate electrostatic repulsion between the cell surface and the antibiotic. The increase in cell surface hydrophobicity which occurred concomitantly with the PMBN-induced permeabilization of the $P$. aeruginosa PAO1 outer membrane to daptomycin is consistent with a lessening of cell surface charge as the result of PMBN binding to the negatively-charged cell surface. That PMBN sensitization of $P$. aeruginosa PAO1 occurred concomitantly with a dramatic decrease in cell surface charge relative to control cells, whereas no sensitization occurred for organisms wherein PMBN failed to affect the overall cell surface charge, confirms the notion that the residual negative charge remaining on otherwise permeabilized outer membranes may preclude daptomycin permeation due to electrostatic repulsion.

The failure of PMBN at relatively high concentrations to cause significant permeabilization of the P. multocida strains to novobiocin, while significant permeabilization of $E$. coli was obtained at a concentration comparable to that used by VAARA and VAARA ${ }^{13)}$ to permeabilize Salmonella typhimurium, suggests that PMBN is not an effective outer membrane permeabilizer for certain nonenteric organisms, particularly in the presence of elevated divalent cation levels. This conclusion is supported by the failure of larger concentrations of PMBN $(51.2 \mu \mathrm{g} / \mathrm{ml})$ to markedly further sensitize Zymomonas mobilis to novobiocin (unpublished data). Alternatively, the inherent susceptibility of naturally-occurring strains of $P$. multocida and $Z$. mobilis to hydrophobic molecules may simply mask further permeabilization of the outer membrane by PMBN.

It is interesting to note that hydrophobic P. multocida variant $\mathrm{P}-1581$ was rendered susceptible to daptomycin with $\mathrm{PMB}^{5)}$ and more hydrophobic by PMBN (Table 2 ), yet did not become less electronegative (Table 2) or susceptible to daptomycin (Fig. 2) as a consequence of growth in the presence of PMBN. Efforts are presently underway to determine if the presence of serotype A encapsulation, which covers an otherwise hydrophobic outer surface in $P$. multocida ${ }^{15)}$ might be responsible for strain variation with regard to susceptibility to the effects of PMB and PMBN on cell surface properties.

\section{Acknowledgments}

This study was supported by a research grant to F.R.C. from 
Lilly Research Laboratories. We are grateful to Lilly Research Laboratories for providing daptomycin, Ms. M. B. HuGHLETr for assistance with cell surface hydrophobicity assays, and Dr. T. R. SHRYOCK for critically reviewing the manuscript.

\section{References}

1) Gilleland, H. E., Jr. \& L. B. Farley: Adaptive resistance to polymyxin in Pseudomonas aeruginosa due to outer membrane impermeability mechanism. Can. J. Microbiol. 28: $830 \sim 840,1982$

2) Leive, L.; S. Telesetsky, W. G. Coleman, Jr. \& D. Carr: Tetracyclines of various hydrophobicities as a probe for permeability of Escherichia coli outer membranes. Antimicrob. Agents Chemother. 25: $539 \sim 544,1984$

3) Brogden, K. A. \& R. B. Rimler: Lysates of turkey-grown Pasteurella multocida: examination of vaccine preparations by electron microscopy. Am. J. Vet. Res. 43: 304 309, 1982

4) Nikaido, H.: Outer membrane of Salmonella typhimurium: transmembrane diffusion of some hydrophobic substances. Biochim. Biophys. Acta. 433: $118 \sim 132,1976$

5) Champlin, F. R. \& M. E. HarT: Cell envelope impermeability to daptomycin in Pseudomonas aeruginosa and Pasteurella multocida. Curr. Microbiol. 21: 367 372, 1990

6) Hart, M. E. \& F. R. Champlin: Susceptibility to hydrophobic molecules and phospholipid composition in Pasteurella multocida and Actinobacillus lignieresii. Antimicrob. Agents Chemother. 32: 1354 1359, 1988

7) Eliopoulos, G. M.; S. Willey, E. Reiszner, P. G. Spitzer, G. Caputo \& R. C. Moellering, Jr.: In vitro and in vivo activity of LY146032, a new cyclic lipopeptide antibiotic. Antimicrob. Agents Chemother. 30: 532 535, 1986

8) Alborn, W. E., Jr.; N. .E. Allen \& D. A. Preston: Daptomycin disrupts membrane potential in growing Staphylococcus aureus. Antimicrob. Agents Chemother. 35: $2282 \sim 2287,1991$

9) Allen, N. E.; W. E. Alborn, Jr. \& J. S. Hobbs, Jr.: Inhibition of membrane potential-dependent amino acid transport by daptomycin. Antimicrob. Agents Chemother. 35: $2639 \sim 2642,1991$

10) LakeY, J. H. \& E. J. A. LeA: The role of acyl chain character and other determinants on the bilayer activity of $\mathrm{A} 21978 \mathrm{C}$ an acidic lipopeptide antibiotic. Biochim. Biophys. Acta. 859: 219 226, 1986

11) VAN der Mei, H. C; A. J. LÉonard, A. H. WeERKamp, P. G. ROUXHET \& H. J. BusSCHER: Surface properties of
Streptococcus salivarius $\mathrm{HB}$ and nonfibrillar mutants: measurement of zeta potential and elemental composition with X-ray photoelectron spectroscopy. J. Bacteriol. 170: $2462 \sim 2466,1988$

12) Ohemeng, K. A.; C. F. Schwender, K. P. Fu \& J. F. BARRETT: DNA gyrase inhibitory and antibacterial activity of some flavones. Bioorg. Med. Chem. Lett. 3: $225 \sim 230,1993$

13) VaAra, M. \& T. VaAra: Polycations sensitize enteric bacteria to antibiotics. Antimicrob. Agents Chemother. 24: $107 \sim 113,1983$

14) Peterson, A. A.; R. E. W. Hancock \& E. J. McGroarty: Binding of polycationic antibiotics and polyamines to lipopolysaccharides of Pseudomonas aeruginosa. $\mathrm{J}$. Bacteriol. 164: 1256 1261, 1985

15) Thies, K. L. \& F. R. Champlin: Compositional factors influencing cell surface hydrophobicity of Pasteurella multocida variants. Curr. Microbiol. 18: 385 390, 1989

16) Darnell, K. R.; M. E. Hart \& F. R. Champlin: Variability of cell surface hydrophobicity among Pasteurella multocida somatic serotype and Actinobacillus lignieresii strains. J. Clin. Microbiol. 25: 67 71, 1987

17) Finegold, S. M. \& W. J. Martin: Determination of susceptibility of bacteria to antimicrobial agents; assay of antimicrobial agents. In Bailey and Scott's Diagnostic Microbiology, 6th ed. pp. 532 557, The C. V. Mosby Co., St. Louis, 1982

18) Rosenberg, M.; D. GUTnick \& E. Rosenberg: Adherence of bacteria to hydrocarbons: a simple method for measuring cell-surface hydrophobicity. FEMS Microbiol. Lett. 9: $29 \sim 33,1980$

19) Watt, J. M.; C. M. Morris, A. George, W. W. Wilson \& F. R. Champlin: Effect of polymyxin $B$ nonapeptide on cell surface charge of permeabilized gram-negative bacteria. Abstracts of Papers of Annu. Meet. South Central Branch Am. Soc. Microbiol., No. 42, p. 18, 1993

20) Tsuchido, T. \& M. TAKano: Sensitization by heat treatment of Escherichia coli K-12 cells to hydrophobic antibacterial compounds. Antimicrob. Agents Chemother. 32: $1680 \sim 1683,1988$

21) Stendahl, O.; L. Edebo, K.-E. Magnusson, C. TAgesson \& S. HJERTÉN: Surface-charge characteristics of smooth and rough Salmonella typhimurium bacteria determined by aqueous two-phase partitioning and free zone electrophoresis. Acta Path. Microbiol. Scand. Sect. B. 85: 334 340,1977

22) CARTER, G. R. \& S. W. Rundell: Identification of type A strains of Pasteurella multocida using staphylococcal hyaluronidase. Vet. Rec. 96: 343, 1975 